\title{
COTREL-DUBOUSSET INSTRUMENTATION AND VERTEBRAL ROTATION IN ADOLESCENT IDIOPATHIC SCOLIOSIS
}

\author{
PETER J. CUNDY, DENNIS C. PATERSON, TERENCE M. HILLIER, ANDREW D. SUTHERLAND, \\ JOHN P. STEPHEN, BRUCE K. FOSTER
}

From Adelaide Children's Hospital and Prince of Wales Children's Hospital, Sydney

\begin{abstract}
We have studied 34 consecutive patients receiving Cotrel- Dubousset instrumentation for a single and flexible thoracic scoliotic curve, evaluating the rib hump deformity from a single CT scan through the apical vertebra of the curve.

Using two measures of rotation we found a mean improvement of $25 \%$ in the rotation of the vertebra after operation. Any, usually minor, deterioration occurred in the first six months postoperatively, and there was no significant further deterioration in 19 patients assessed over two years after surgery.

Cotrel-Dubousset instrumentation can produce a significant correction of vertebral rotation and of the associated rib hump deformity.
\end{abstract}

During the last 25 years surgical treatment of scoliosis has been able to achieve significant corrections in fixed lateral curvature of the spine, but less correction of vertebral rotation has been possible. Weatherley et al (1987) have shown that $64 \%$ of patients with scoliosis treated by Harrington compression and distraction instrumentation achieved little improvement in their rib hump deformity, despite good correction of lateral curvature, as determined by measurement of the Cobb angle (Cobb 1948). It has been claimed that CotrelDubousset instrumentation provides a significant correction of rotation and a marked reduction in the cosmetic rib hump deformity.

Cotrel-Dubousset spinal instrumentation was first used at the Adelaide Children's Hospital in mid-1986 and later at the Prince of Wales Children's Hospital, Sydney. We have made a prospective study of the correction of vertebral rotation achieved by this instrumentation.

P. J. Cundy, FRACS, Research Fellow

D. C. Paterson, MD, FRCS, FRACS, Director and Chief Orthopaedic Surgeon

T. M. Hillier, FRACS, Senior Visiting Orthopaedic Surgeon

A. D. Sutherland, FRACS, Senior Visiting Orthopaedic Surgeon

B. K. Foster, MD, FRACS, Deputy Director

Department of Orthopaedic Surgery, Adelaide Children's Hospital, 72

King William Road, North Adelaide, South Australia 5006, Australia.

J. P. Stephen, FRACS, Senior Visiting Orthopaedic Surgeon

Department of Orthopaedic Surgery, Prince of Wales Children's Hospital, Sydney, New South Wales, Australia.

Correspondence should be sent to Sir Dennis Paterson.

(C) 1990 British Editorial Society of Bone and Joint Surgery $0301-620 X / 90 / 4146 \$ 2.00$

J Bone Joint Surg [ Br] 1990; 72-B: 670-4.

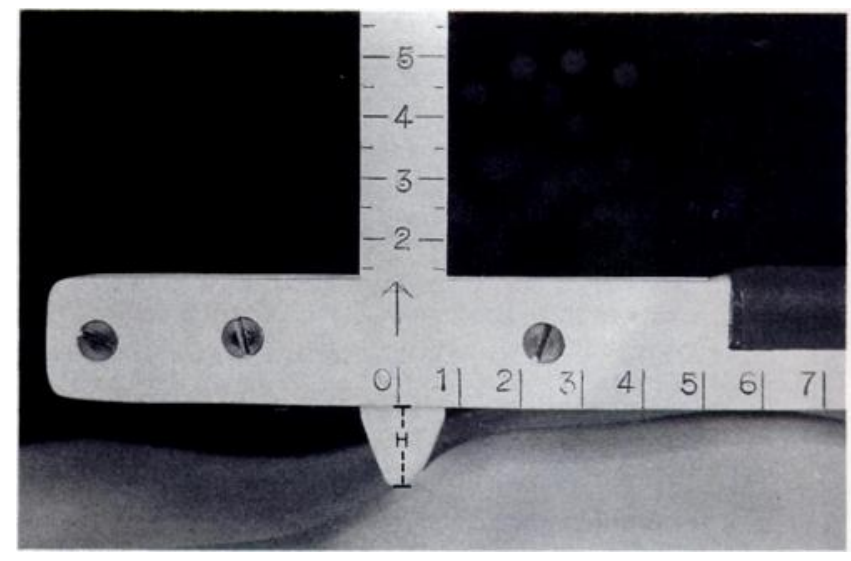

Fig. 1

The modified scoliometer used to measure the rib hump deformity before and after operation (see text).

\section{PATIENTS AND METHODS}

Of the first 40 consecutive cases at either centre, 34 were treated for a single and flexible thoracic scoliotic curve: these cases have been studied in detail.

Pre-operative assessment. Each patient gave a complete history; physical examination paid particular attention to the extent of the rib hump and the neurological status. Radiographic studies included standing anteroposterior and lateral films and also anteroposterior films with the patient bending fully to the right and left to determine the mobility of the spinal deformity. The height of the rib hump was measured and a single CT slice was made through the apical vertebra with the patient in the supine position to assess vertebral rotation and the rib cage deformity. Pre-operative somatosensory evoked potential 
recordings were made in preparation for intra-operative spinal cord monitoring.

Two patients with large thoracic curves had preoperative halo-femoral traction for two weeks, after which further radiographs were made and CT scans were repeated in traction before operation. Traction was not continued during or after the operation. Cotrel-Dubousset instrumentation and posterior spinal fusion was performed by one of four surgeons, using a standard technique, continuous spinal cord monitoring was provided and the Stagnara 'wake-up' test was used during the operation.

Postoperative assessment. Each patient had standing anteroposterior and lateral radiographs and a single CT slice through the same apical vertebra. The height of the rib hump was evaluated when the patient could bend forward far enough for this to be compared with the preoperative evaluation. All complications were recorded, and patients were assessed at regular intervals. Nineteen patients were reassessed at least two years after surgery for any change in the correction of the deformity.

Rib hump evaluation. The rib hump was measured by a modification of the method suggested by Thulbourne and Gillespie (1976). In the forward bending position, a vertical movable rod was placed upon the spinous process of the apical vertebra and a horizontal beam incorporating a spirit level was balanced on the rib hump (Fig. 1). The maximum height $(\mathrm{H})$ of the rib hump was recorded. CT scans. At first, the CT scans were assessed using the guidelines of Aaro and Dahlborn (1981a,b), but this technique was simplified to provide more reliable data. We used only precise bony landmarks and did not measure the rib hump from the scans, since this was found to be inaccurate.

The landmarks were the middle of the sternum, the centre of the posterior neural arch, and the axis of the vertebra (Fig. 2). The angle formed between the midline of the torso (a line between the centre of the posterior arch and the middle of the sternum) and the axis of the vertebra was recorded as the vertebral rotation relative to the midline (RAml). This RAml angle was measured in the pre- and postoperative CT scans independently by three persons on at least two separate occasions in order to assess the interobserver and intra-observer error.

The vertebral rotation was also measured as the angle between a perpendicular and the axis of the vertebra (Fig. 3), and recorded as the RAsagittal (Aaro and Dahlborn 1981a,b).
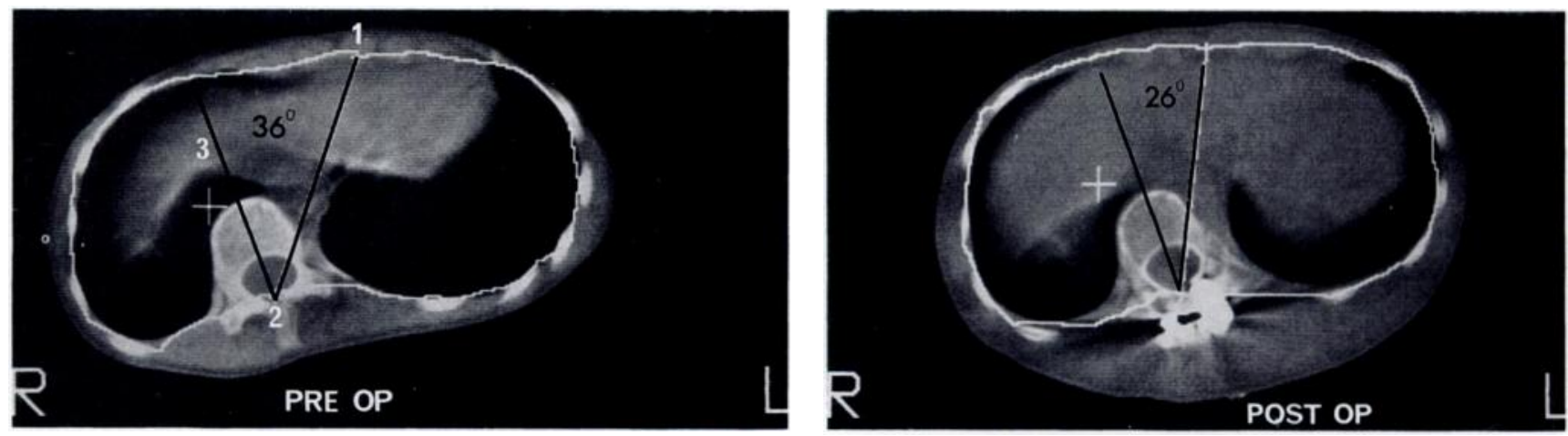

Fig. 2

Rotation relative to the midline (RAml) was measure pre- and postoperatively using the middle of the sternum (1), the centre of the posterior neural arch (2) and the axis of the vertebra (3).
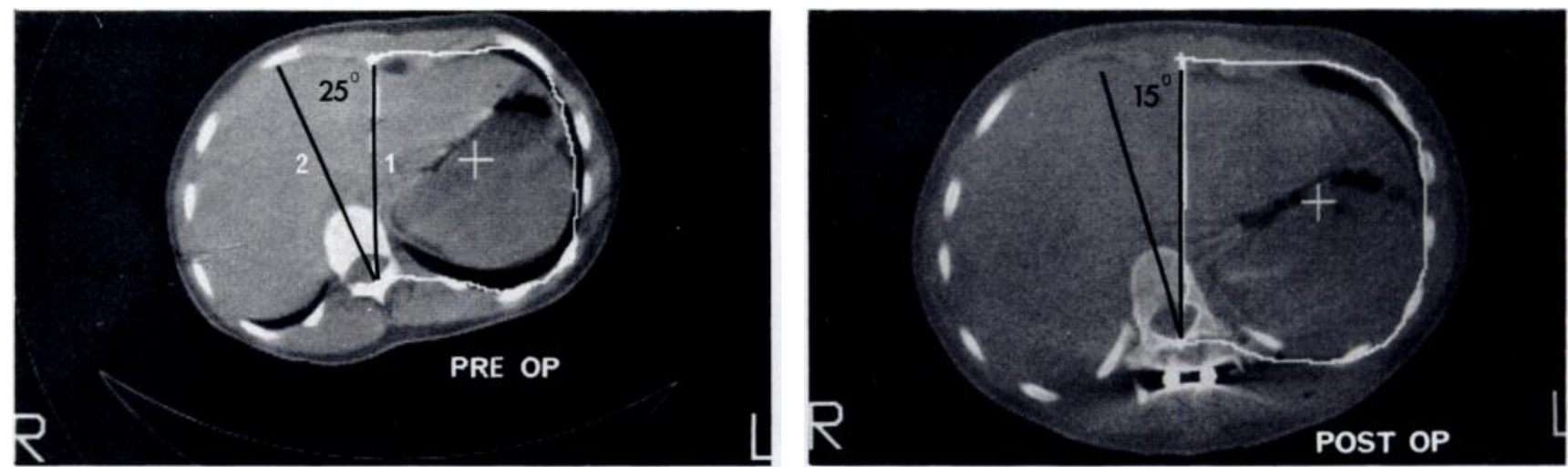

Fig. 3

Rotation relative to a perpendicular (RAsagittal) was measured pre- and postoperatively using a perpendicular (1) and the axis of the vertebra (2). 

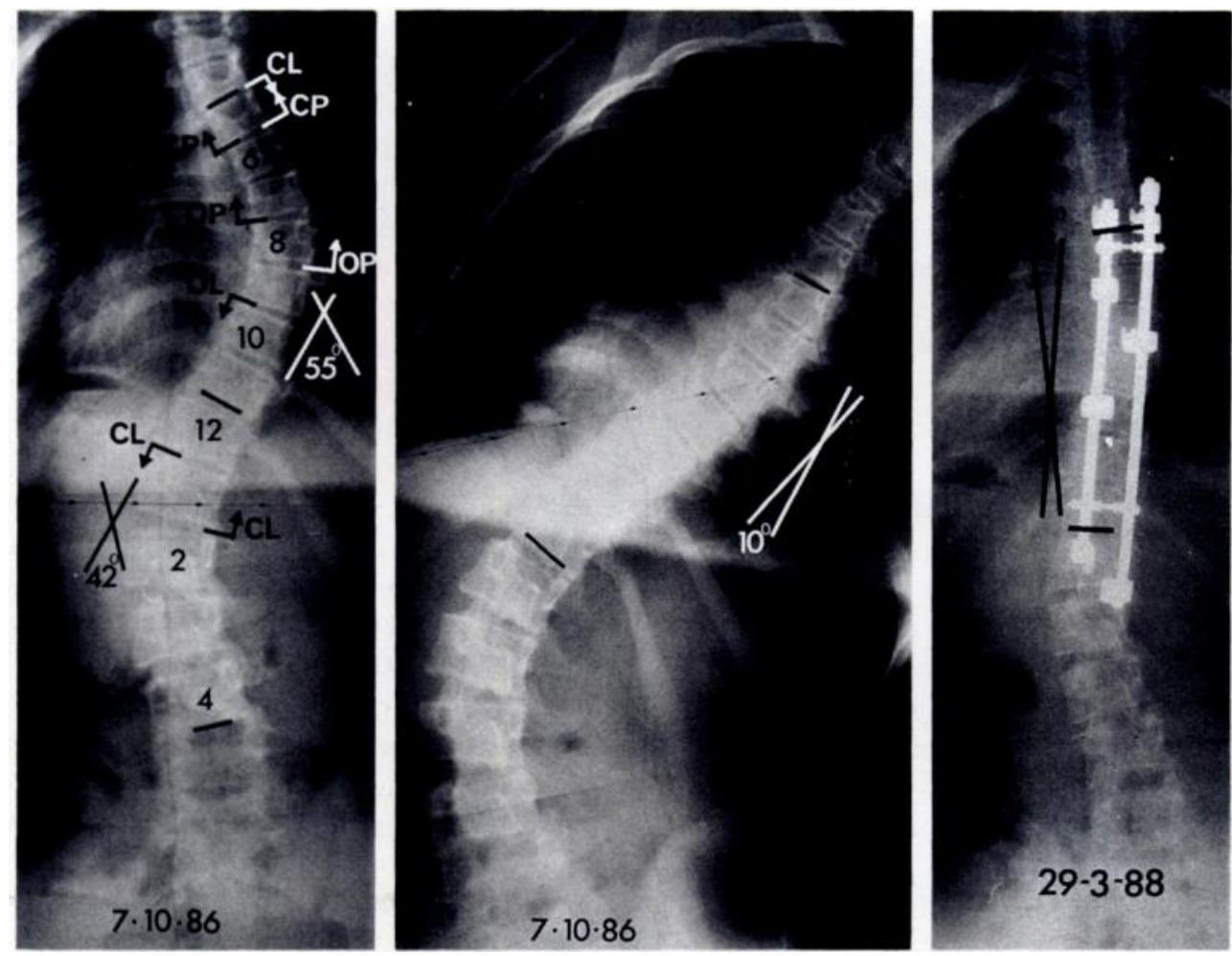

Fig. 4

Radiographs of a patient with an idiopathic scoliosis, standing, right lateral bending and postoperatively. Hook placements are indicated by $\mathrm{CP}=$ closed pedicle, $\mathrm{CL}=$ closed lamina, $\mathrm{OP}=$ open pedicle, $\mathrm{OL}=$ open lamina.

\section{RESULTS}

Cobb angle. The mean pre-operative Cobb angle was $48.1^{\circ}$ (range $30^{\circ}$ to $82^{\circ}$ ) and the mean postoperative angle was $16.2^{\circ}$ (range $2^{\circ}$ to $45^{\circ}$ ). This represents a $67 \%$ correction (Figs 4 and 5). This correction was further assessed in relation to the mobility of the curve on preoperative bending films. A mean correction of $8^{\circ}$ (range $0^{\circ}$ to $30^{\circ}$ ) beyond that in lateral bending film had been obtained.

CT scans. The mean inter and intra-observer errors of measurement of both RAml and RAsagittal angles was $2.5^{\circ}$. Measurements on the CT scans showed a preoperative mean RAml of $29.1^{\circ}$ (range $15^{\circ}$ to $50^{\circ}$ ) and a postoperative mean of $21^{\circ}$ (range $7^{\circ}$ to $40^{\circ}$ ). This indicates a $24 \%$ derotation of the vertebra. The mean pre-operative RAsagittal angle was $14^{\circ}$ (range $4^{\circ}$ to $27^{\circ}$ ) against a postoperative mean of $10.4^{\circ}$ (range $0^{\circ}$ to $23^{\circ}$ ), indicating a $26 \%$ improvement in rotation.

The RAsagittal angle increased in four patients, by $1^{\circ}$ to $6^{\circ}$ (mean $1.2^{\circ}$ ) whereas the RAml angle decreased in all patients.

Rib cage. The contour of the rib cage was seen to improve in all CT scans, with the vertebral body assuming a more central position within the thorax. This was evident when the soft-tissue shadows of the CT scans were superimposed (Fig. 6). The mean height of the rib hump decreased from $17 \mathrm{~mm}$ (range 10 to 28 ) to $4 \mathrm{~mm}$ (range 0 to 15$)$.
Follow-up studies. Ten patients were assessed from 12 to 20 months after their surgery (mean 14.4 months). The mean loss of Cobb angle correction was $3.5^{\circ}$ (range $+2^{\circ}$ to $-11^{\circ}$ ). Both the CT scan measurements and the hump height also showed a slight loss of correction. The RAml angle increased by a mean of $1.4^{\circ}$ (range $-5^{\circ}$ to $+9^{\circ}$ ) being reduced in four and increased in six cases. The rib hump deformity increased measurably in only one patient by $7.5 \mathrm{~mm}$.

However, the 19 patients assessed at 24 to 36 months (mean 30) showed a mean loss of only $2.6^{\circ}$ in this period. The improved figure was due to better mean corrections obtained in the extra nine cases. In two patients, the Cobb angle decreased by $2^{\circ}$ while in the other 17 , there was an increase up to $11^{\circ}$.

These findings suggest that correction obtained at operation did not deteriorate significantly over a two- to three-year period. No loss of correction was seen later than six months postoperatively. These results compare favourably with the correction maintained with Harrington instrumentation (Nash and Moe 1969).

\section{DISCUSSION}

It has been shown that Harrington compression and distraction instrumentation allows a rib hump to increase with time: Weatherley et al (1987), reported a series with a mean loss of Cobb angle of $9^{\circ}$ in the first year. Our 

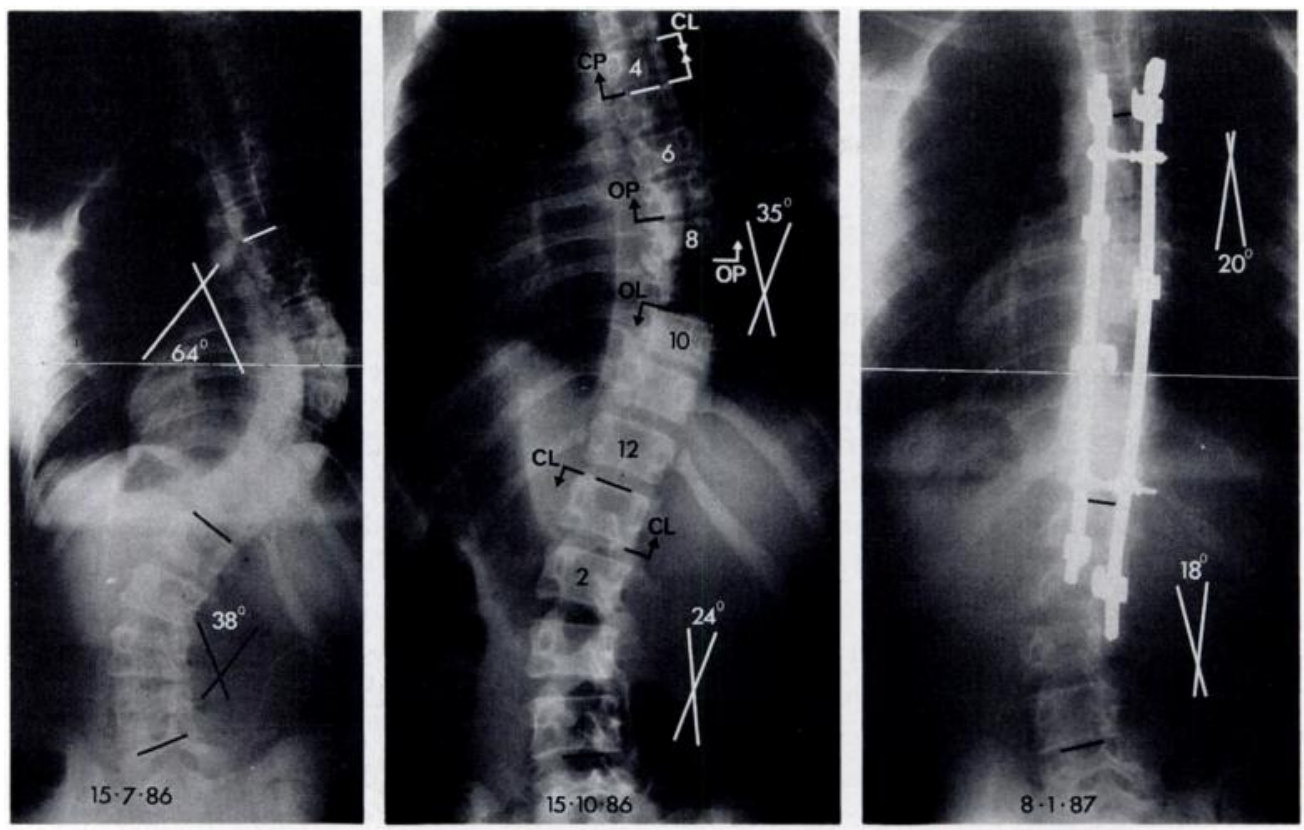

Fig. 5

Radiographs of a patient with an idiopathic thoracic scoliosis and secondary lumbar scoliosis before treatment, after two weeks in halo-femoral traction and postoperatively.

initial experience with Cotrel-Dubousset instrumentation has shown that the correction obtained at operation is maintained better than that obtained with Harrington rod instrumentation. We attribute this to the segmental nature of the instrumentation.

Correction of the rib hump deformity is one of the main aims of surgery for adolescent idiopathic scoliosis; failure to achieve this is disappointing for both patient and surgeon. Our study has shown that a good clinical correction of the rib hump can be achieved and maintained for at least two years by derotation of the vertebral column with the Cotrel-Dubousset instrumentation. This technique has also significantly corrected the lordoscoliotic deformity, and has produced a kyphotic thoracic spine (Deacon, Flood and Dickson 1984; Dickson et al 1984; Dickson and Archer 1987).

It is apparent that minor loss of correction occurs in the first months postoperatively and does not deteriorate further, indicating that a sound fusion has then been achieved. Hence, the incidence of pseudarthrosis in the fusion has also decreased markedly.

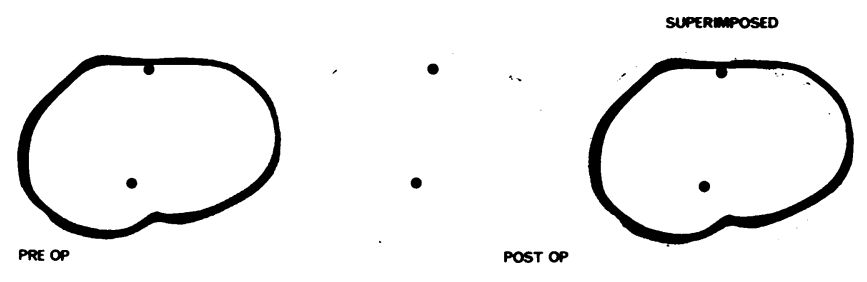

Fig. 6

Superimposed CT scans show the improvement in contour of the rib cage.
Measurement of vertebral rotation is difficult without the use of CT scans. Nash and Moe (1969) described a method of determining vertebral rotation using standard anteroposterior radiographs and estimating the position of the pedicles across the vertebra. This method has been assessed by Drerup (1985) who suggested modifications in the measurement points to allow more accurate determination of the angle of rotation. The pedicles are often obscured by the longitudinal metal rods of the instrumentation; postoperative measurements may not be possible. In addition, measurements of rotation from anteroposterior radiographs in mild to moderate scoliosis are often unreliable due to variations in the angle and projection of the pedicles in the preoperative and postoperative radiographs. Another diffculty is with variation in the morphology of the pedicles (Benson, Schultz and Dewald 1976).

By contrast, CT scanning allows accurate localisation of the three bony landmarks both before and after operation. A single CT cut is performed through the centre of the apical vertebra; the body contour outline at that level is then available for standard assessment. The sternum and the posterior neural arch are readily identifiable, so the axis of the vertebra itself is the main source of observer error, especially where the vertebra itself is asymmetrical. However, in our series of idiopathic cases there was minimal asymmetry of the bodies and accurate plotting of the axis was possible.

The RAsagittal angle, using a perpendicular, may be inaccurate because of the variable position of the patient on the CT table. The postoperative reduction of the rib hump will allow the supine patient to roll towards the convexity of the spinal deformity, thereby increasing 
the apparent RAsagittal angle and giving a false impression of an increase in rotational deformity. In our series, the RAsagittal angle gave lower mean values than the RAml angle both before and after operation but did show a similar percentage correction of rotation.

However, the RAsagittal angle increased in four patients while the RAml angle decreased in all patients. This could be due to the reduction of the rib hump changing the position of the patient, as discussed above, or to the effect on the RAml angle of centralising the vertebra within the rib cage. After operation, the vertebrae lie more directly behind the sternum; this alters the central point of the measurements and appears to be the more likely explanation. Thus, the RAml angle measures two factors: vertebral rotation and the displacement of the vertebrae towards the midline. Measurements incorporating these factors have previously been reported by Banzer, Risch and Wegener (1980).

The contour of the rib cage improved in all cases. It was not possible to quantify this, but superimposing the pre-operative and postoperative CT scans, centring them on a line between the posterior neural arch and the sternum, showed good correction (Fig. 6).

Previous workers (Goldstein 1973; Thulbourne and Gillespie 1976) reported that the rib hump deformity had no direct relationship to the degree of vertebral rotation nor to the lateral curve of the spinal deformity. We found, however, a significantly positive rank correlation between the rib hump deformity, the lateral curve and vertebral rotation, particularly for the pre-operative measurements. The pre-operative Cobb angle and RAml angle had a Spearman's rank correlation coefficient of 0.66 , significant at the $1 \%$ level (Beyer 1987). This significance was not changed by the variation of results in respect of inter and intra-observer errors. We suggest that the Nash and Moe (1969) method of measurement of vertebral rotation used by previous workers failed to provide data accurate enough to reveal these relationships. Our findings result from the better method of assessment of vertebral rotation.

Conclusions. Evaluation of vertebral rotation and rib cage deformity by CT scans has established that CotrelDubousset instrumentation produced a significantly better correction of cosmetic deformity in idiopathic adolescent scoliosis than previous techniques. Dickson and Archer (1987) have emphasised the three-dimensional nature of the deformity of idiopathic scoliosis and that instrumentation which addresses this fact is essential for adequate correction.

We acknowledge the help that we have received from Thomas B. van Dulken, Senior Tutor, Department of Mathematics of the Flinders University, Adelaide and David M. Dickison, Orthopaedic Registrar at the Prince of Wales Children's Hospital, Sydney.

We wish to thank Mr Mark Stevens, Department of Photography, at the Adelaide Children's Hospital, for his help with the illustrations.

No benefits in any form have been received or will be received from a commercial party related directly or indirectly to the subject of this article.

\section{REFERENCES}

Aaro S, Dahlborn M. Estimation of vertebral rotation and the spinal and rib cage deformity in scoliosis by computer tomography. Spine $1981 \mathrm{a} ; 6: 460-7$.

Aaro S, Dahlborn M. The longitudinal axis rotation of the apical vertebra, spinal, and rib cage deformity in idiopathic scoliosis studied by computer tomography. Spine 1981 b; 6:567-72.

Banzer D, Risch WD, Wegener OH. Eine neue Methode zur Messung des Rotationswinkels der Wirbelskoliose. ROEFO 1980; 132:4035. (Eng. abstr.)

Benson DR, Schultz AB, Dewald RL. Roentogenographic evaluation of vertebral rotation. J Bone Joint Surg [ Am] 1976; 58-A:1125-9.

Beyer WH, ed. CRC handbook of tables for probability and statistics. 2nd ed. Boca Raton, Florida: CRC Press, 1987.

Cobb JR. Outline for the study of scoliosis. Am Acad Orthop Surg Instr Course Lect 1948; 5:261-75.

Deacon P, Flood BM, Dickson RA. Idiopathic scoliosis in three dimensions: a radiographic and morphometric analysis. $J$ Bone Joint Surg [Br] 1984; 66-B:509-12.
Dickson RA, Lawton JO, Archer IA, Butt WP. The pathogenesis of idiopathic scoliosis. J Bone Joint Surg [Br] 1984; 66-B:8-15.

Dickson RA, Archer IA. Surgical treatment of late-onset idiopathic thoracic scoliosis: the Leeds procedure. J Bone Joint Surg [Br] 1987; 69-B:709-14.

Drerup B. Improvements in measuring vertebral rotation from the projections of the pedicles. J Biomech $1985 ; 18: 369-78$.

Goldstein LA. The surgical treatment of idiopathic scoliosis. Clin Orthop 1973; 93:131-57.

Nash CL Jr, Moe JH. A study of vertebral rotation. J Bone Joint Surg [Am] 1969; 51-A :223-9.

Thulbourne T, Gillespie R. The rib hump in idiopathic scoliosis: measurement, analysis and response to treatment. $J$ Bone Joint Surg [Br] 1976; 58-B:64-71.

Weatherley CR, Draycott V, O'Brien JF, et al. The rib deformity in adolescent idiopathic scoliosis: a prospective study to evaluate changes after Harrington distraction and posterior fusion. $J$ Bone Joint Surg [ Br] 1987; 69-B:179-82. 